\title{
CONCURRENCE IMPARFAITE ET DISCRIMINATION SUR LE MARCHE DU TRAVAIL
}

Clémence Berson ${ }^{1}$

Les modèles de discrimination sur le marché du travail reproduisent difficilement sa persistance sans faire d'hypothèses fortes. Cela conduit de surcroît à une ségrégation des travailleurs selon leurs caractéristiques non productives. Dans cet article, le modèle se sert de l'oligopsonie et de l'hétérogénéité des préférences des travailleurs pour obtenir une persistance de la discrimination. Les entreprises embauchent les deux types de travailleurs et offrent un salaire plus faible aux travailleurs discriminés. Par conséquent, l'existence de discrimination sur le marché permet aux entreprises n'ayant pas de goût pour la discrimination de faire des profits non nuls. Elles n'ont donc pas d'incitation à faire sortir les entreprises discriminantes du marché.

\section{IMPERFECT COMPETITION AND DISCRIMINATION ON THE LABOR MARKET}

Discrimination models have difficulties to reproduce a persistent discrimination without assuming that prejudiced firms are more productive and results lead to workers' segregation. The model uses oligopsony and heterogeneity of workers' preferences to obtain a persistent discrimination. Firms hire both types of workers and pay a lower wage to the workers discriminated against. Consequently, the existence of discrimination allows a non-zero profit for unprejudiced firms and they have also no incentives to push out prejudiced firms.

Classification JEL : J42, J71, L13.

\section{INTRODUCTION}

La discrimination a été introduite en économie par Becker [1957]. Elle consiste à traiter de manière différente deux personnes aux caractéristiques

\footnotetext{
${ }^{1}$ Paris School of Economics - Université Paris 1 Panthéon-Sorbonne et Ecole Polytechnique. Contact : MSE, 106-112, bd de l'Hôpital, 75013 Paris, clemence.berson@univparis1.fr. Je remercie Pierre Cahuc ainsi que les participants du Séminaire IMA de Paris 1 et du congrès de l'AFSE 2010.
} 
productives identiques mais ayant une caractéristique non productive observable différente. Becker considère que certains employeurs, collègues ou clients ont un goût particulier pour la discrimination. Par conséquent, les membres du groupe discriminé reçoivent un salaire plus faible pour compenser ce désagrément. Arrow [1973] et Phelps [1972] développent la discrimination statistique en alternative à la vision de Becker. Elle est fondée sur les croyances. Si le salarié appartient au groupe de l'employeur, ce dernier connaît sa véritable productivité. Si le salarié appartient au groupe discriminé, que l'employeur ne connaît pas, ce dernier pense qu'il est en moyenne moins productif que son propre groupe et le paye alors selon cette moyenne et non selon sa productivité réelle. Dans les deux cas, la discrimination tend à disparaître dans le long terme. Dans le cas de Becker, la concurrence implique des profits nuls, ce qui signifie que les entreprises discriminantes, moins compétitives que les autres à cause de coûts de production plus importants, doivent sortir du marché. Leur persistance montre qu'elles ont des rentes ou qu'elles sont plus productives que les autres entreprises. Dans le cas de la discrimination statistique, les employeurs apprennent la réelle productivité de leurs travailleurs avec l'expérience dans l'entreprise et le différentiel de salaire disparaît. Cet article utilise la discrimination à la Becker, mais peut également être utilisé pour la discrimination statistique.

Des études montrent que la discrimination diminue avec la concurrence, mais ne disparaît pas (Black et Strahan [2001], Hellerstein, Neumark et Troske [2002]). L'existence d'imperfections sur le marché des biens justifie cette persistance par la présence de rentes permettant de satisfaire le goût des employeurs pour la discrimination sans sortir du marché. La baisse de ces rentes oblige les employeurs à diminuer le salaire de leurs employés sur-payés afin de rester compétitifs et permet donc de diminuer les différentiels de salaire. Mais la discrimination ne repose pas entièrement sur l'imperfection du marché des biens. Elle ne respecte pas non plus la théorie de la concurrence sur le marché du travail. En effet, un travailleur doit être payé à sa productivité marginale et par conséquent deux travailleurs ayant les mêmes caractéristiques productives doivent recevoir le même niveau de salaire. La présence de discrimination viole cette condition puisqu'elle implique qu'un travailleur reçoive un salaire plus faible du fait d'une caractéristique non productive. Dans cet article, c'est cette dernière caractéristique que l'on exploite.

Peu de modèles théoriques utilisent un modèle basé sur le goût pour la discrimination car les modèles classiques ne peuvent l'expliquer sans hypothèses fortes. L'introduction de frictions sur le marché du travail permet d'établir un cadre de concurrence imparfaite. Le modèle de recherche d'emploi développé par Black [1995] aboutit à un différentiel de salaire durable mais il fait l'hypothèse d'une plus grande productivité des entreprises discriminantes afin de les conserver sur le marché. Il en résulte une ségrégation des travailleurs selon leur groupe d'appartenance. Les modèles d'appariement et de recherche font ce type d'hypothèses afin de justifier la persistance de la discrimination. De plus, les modèles d'appariement se focalisent sur les négociations de salaire ex ante, ce qui est mal adapté aux salaires peu élevés qui sont le plus souvent postés. 
Cet article décrit un modèle basé sur le goût pour la discrimination où les travailleurs ne sont pas payés à leur productivité marginale. En effet, les individus prennent en compte des caractéristiques non-salariales des emplois comme la distance physique (utilisée dans l'article), des caractéristiques sociales de l'entreprise, les conditions de travail ou même la présence de frictions informationnelles pour choisir leur emploi. Cette hétérogénéité des préférences des travailleurs implique une élasticité imparfaite de l'offre de travail au salaire. Les employeurs exploitent cette imperfection et fixent alors les salaires. L'hétérogénéité des préférences leur confère donc un pouvoir de marché.

Les principales contributions de cet article sont que les entreprises n'ayant pas de goût pour la discrimination discriminent la minorité et que les entreprises non discriminantes n'ont pas intérêt à évincer les entreprises discriminantes du marché car leur présence leur permet de faire des profits non nuls. Afin d'obtenir ce modèle d'oligopsonie, on utilise un modèle d'hétérogénéité des préférences basé sur le modèle de Salop [1979]. Il permet d'obtenir un différentiel de salaire durable entre la majorité et la minorité sans ségrégation. A ma connaissance, ce modèle est pour la première fois appliqué à des problématiques de discrimination sur le marché du travail. Thisse et Zénou [1995], Wauthy et Zénou [1999] et Hamilton, Thisse et Zénou [2000] se servent de ce modèle pour étudier les politiques d'emploi et de formation continue. Bhaskar et To [2003] l'emploient pour modéliser la dispersion des salaires et enfin, Baskar, Manning et To [2002] font une revue des emplois possibles de ce modèle en économie du travail. Ces derniers exposent l'intuition de cet article en prenant l'exemple de deux entreprises : une discriminante et l'autre n'ayant pas de goût pour la discrimination. La présence d'un différentiel de salaires entre les deux populations provient des fonctions de réaction utilisées par les deux entreprises pour fixer leurs salaires.

La première partie de cet article présente les bases du modèle. La seconde partie se focalise sur le cas de quatre entreprises afin de mieux étudier l'impact de la discrimination. Les effets de la concurrence sur la discrimination sur le marché du travail sont étudiés dans la troisième partie. Enfin, la dernière partie conclut.

\section{LE MODELE}

Le marché est représenté par une ville circulaire telle que Salop [1979] l'a décrite. $n$ entreprises sont réparties uniformément sur le cercle et la distance entre deux entreprises est donc $\frac{1}{n}$. Le marché du travail est composé d'un continuum de travailleurs de deux types: A et B. Les deux populations de travailleurs ne diffèrent que par une caractéristique observable non productive. Les B représentent une part $\gamma$ de la population active et les deux types de travailleurs sont répartis uniformément sur le marché circulaire. Les travailleurs doivent se rendre dans une entreprise $i$ pour avoir un emploi. Tous les déplacements se font le long du cercle et chaque travailleur doit parcourir une distance $x$ pour aller travailler. Il dépense ainsi $t x$ pour le trajet. L'introduction 
d'une distance au lieu de travail est équivalente à introduire de l'hétérogénéité dans les préférences des travailleurs. La distance peut être physique, mais elle peut aussi être interprétée comme les caractéristiques non salariales de l'emploi (type de contrat, nombre d'heures travaillées...), comme des caractéristiques de l'entreprise elle-même (taille, politiques environnementale ou sociale...) ou bien comme des frictions informationnelles (distance à l'information...). Cela signifie que les emplois ne sont pas parfaitement substituables et donc que l'élasticité de l'offre de travail au salaire n'est pas parfaite. Les entreprises sont en situation d'oligopsonie sur le marché du travail. L'hétérogénéité entraîne un pouvoir de marché en faveur des entreprises qui peuvent alors fixer leurs salaires et discriminer leurs salariés.

Chaque entreprise emploie uniquement le travail pour produire un bien vendu au prix $p$ sur un marché compétitif. Chaque travailleur produit une unité de bien sur la période. Afin de simplifier le modèle, il n'y a pas de libre entrée ou sortie sur le marché. On distingue deux types d'entreprises : celles ayant un goût pour la discrimination et celle n'en ayant pas. Les premières représentent une part $\eta$ du nombre total d'entreprises. Soit $d$ le coût psychologique de l'employeur lorsqu'il emploie un travailleur B. Ce coût est nul pour les employeurs n'ayant pas de goût pour la discrimination.

Soit $w_{j i}$ le salaire offert par l'entreprise $i$ à un travailleur du groupe $j$. Afin de conserver un cadre simple, on suppose que le salaire de réservation est normalisé à zéro. Le salaire net est le salaire offert auquel on retire les frais de transport, $w_{j i}-x t$ pour un travailleur $j$ de l'entreprise $i$. Un travailleur accepte l'emploi uniquement si le salaire net est supérieur au salaire de réservation, donc s'il est positif. Dans le cas contraire, il préfère être au chômage. Le choix de l'entreprise se fait en comparant les salaires nets : si $w_{j i}-t x>w_{j k}-t\left(\frac{1}{n}-x\right)$ quel que soit $k$ alors l'individu va offrir sa force de travail à $i$.

Les employeurs maximisent leur fonction d'utilité notée $U_{i}\left(\Pi_{i}, L_{A i}, L_{B i}\right)$ et qui dépend à la fois du profit de leur entreprise et du type des travailleurs embauchés.

Les employeurs recrutent des travailleurs tant qu'ils génèrent du profit. Lorsque la vente du produit ne suffit plus à compenser les dépenses de salaire, les employeurs cessent d'embaucher. En effet, si une entreprise veut embaucher des travailleurs supplémentaires, elle doit augmenter le salaire pour que des travailleurs plus éloignés puissent être intéressés. L'utilité d'un employeur est donc la suivante :

$$
U_{i}=p\left(L_{A i}+L_{B i}\right)-w_{A i} L_{A i}-\left(w_{B i}+d_{i}\right) L_{B i}
$$

où $d_{i}=d$ si l'entreprise $i$ a un goût pour la discrimination et $d_{i}=0$ autrement, et $L_{j i}$ est le nombre de travailleurs $j$ employés. On rappelle que $d$ peut être de la discrimination statistique. Dans ce cas, l'employeur discriminant suppose que les travailleurs B sont en moyenne moins productifs que les travailleurs A, car il connaît peu cette population. La productivité des B est alors en moyenne $1-\frac{p}{w}$ inférieure à celle des $\mathrm{A}$ et le salaire correspondant est de $w-d$. Les résultats qui vont suivre seront identiques car le modèle est 
statique, l'employeur ne peut donc pas apprendre la productivité réelle des B.

Dans ce modèle, on suppose que le marché du travail est couvert. En effet, dans le cas contraire, les entreprises sont en monopsone ce qui retire l'intérêt de l'oligopsonie. Pour prendre le chômage en compte, il faut introduire un salaire de réservation hétérogène. Le modèle est alors trop complexe pour notre étude et doit faire l'objet d'un autre article sur le sujet.

\section{LE CAS DE QUATRE ENTREPRISES}

Dans cette partie, nous focalisons sur le cas de quatre entreprises. Cela permet de mieux comprendre le fonctionnement du modèle et les résultats sont généralisables à $n$ entreprises.

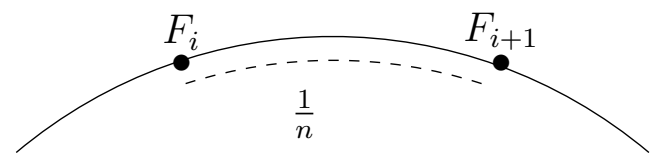

Lorsque $\left|w_{j i}-w_{j i+1}\right| \leq \frac{t}{4}$ pour tout $i$, les travailleurs situés entre $i$ et $i+1$ n'ont aucune incitation à travailler pour une autre entreprise que $i$ ou $i+1$. Ils offrent donc leur travail en comparant les salaires nets de ces deux entreprises. Un individu décide donc de travailler pour l'entreprise $i$ si $w_{j i}-x t>w_{j i+1}-$ $\left(\frac{1}{4}-x\right) t$. Le travailleur situé à $\tilde{x}=\frac{1}{2 t}\left(\frac{t}{4}+w_{j i}-w_{j i+1}\right)$ est indifférent entre travailler pour $i$ ou $i+1$. L'arbitrage est identique pour les individus situés entre $i$ et $i-1$ et l'offre de travail est donc symétrique. Comme les travailleurs sont répartis uniformément sur le cercle, on a une proportion $\gamma$ de $\mathrm{B}$ et $1-\gamma$ de A qui offrent leur travail à $i$ :

$$
L_{i}=\gamma \frac{1}{t}\left(\frac{t}{4}+w_{B i}-\bar{w}_{B i}\right)+(1-\gamma) \frac{1}{t}\left(\frac{t}{4}+w_{A i}-\bar{w}_{A i}\right)
$$

où $\bar{w}_{j i}=\frac{1}{2}\left(w_{j i-1}+w_{j i+1}\right)$ est la moyenne des salaires offerts par les voisines directes de $i$. Le salaire dépendant du groupe auquel le travailleur appartient, on distingue l'offre des $\mathrm{B}$ (première partie de l'équation) de celle des A (seconde partie).

Les employeurs maximisent leur utilité et obtiennent leur(s) fonction(s) de réaction. En substituant l'équation d'offre de travail dans l'utilité de l'employeur et en résolvant la condition de premier ordre, les salaires optimaux de l'entreprise $i$ sont :

$$
\begin{aligned}
w_{A i} & =\frac{1}{2}\left(p-\frac{t}{4}+\bar{w}_{A i}\right) \\
w_{B i} & =\frac{1}{2}\left(p-\frac{t}{4}+\bar{w}_{B i}-d_{i}\right)
\end{aligned}
$$


Le salaire des A n'est pas affecté par la discrimination et il est identique quel que soit l'entreprises $w_{A i}=p-\frac{t}{4}$. En effet, les fonctions de réaction des salaires des A sont toutes identiques. On remarque que le salaire n'est pas égal à la productivité du travailleur à cause de la présence des coûts de transport. Une dispersion des salaires est possible si on considère que la productivité des entreprises est hétérogène, comme l'ont fait Bhaskar et To [2003]. Ce n'est pas le cas dans cet article afin de conserver un cadre simple et d'observer au mieux les variations de salaire dues à la discrimination.

La fonction de réaction des entreprises non discriminantes est identique quel que soit le groupe du travailleur car $d_{i}=0$. Le différentiel de salaire va apparaître par le biais du salaire offert par les entreprises voisines : une entreprise discriminante va offrir un salaire plus faible aux B qui se répercute dans les salaires des entreprises non-discriminantes. Ainsi, la présence d'une entreprise discriminante sur le marché a un effet négatif sur les salaires de tous les B, même s'ils sont employés par une entreprise non discriminante. Par conséquent, un différentiel de salaire est observable entre A et B dans chaque entreprise. Conformément à l'intuition, la proportion d'entreprises discriminantes sur le marché a un impact positif sur le différentiel de salaire moyen entre les $A$ et les $B$. Le différentiel moyen lorsqu'il n'y a qu'une seule entreprise discriminante sur le marché est de $\frac{1}{4} d$. Il atteint $\frac{1}{2} d$ lorsque deux entreprises discriminantes sont présentes sur le marché. Il passe à $\frac{3}{4} d$ en présence de trois entreprises ayant un goût pour la discrimination.

Afin d'étudier l'impact de la répartition des entreprises sur le cercle, on suppose pour la suite que $\eta=0,5$. Les entreprises 1 et 2 sont supposées non discriminante et les entreprises 3 et 4 ont un goût pour la discrimination. Deux configurations sont possibles : soit les entreprises identiques sont face à face, soit elles sont côte à côte (cf figure 1).
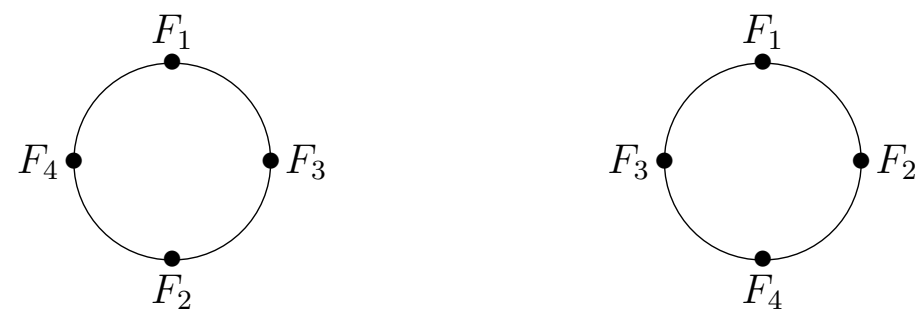

Figure 1: Marché à quatre entreprises. A gauche les entreprises discriminantes alternant avec les non discriminantes, à droite les entreprises identiques côte à côte.

Le salaire des A est identique quelque soit la répartition des entreprises. Cependant, le salaire des $\mathrm{B}$ diffère avec la configuration des entreprises. En effet, si $d \leq \frac{5}{6} t$, les salaires d'équilibre des B sont respectivement pour la configuration 1 et 2 : 


$$
\left\{\begin{array} { l } 
{ w _ { B 1 } = w _ { B 2 } = p - \frac { t } { 4 } - \frac { 1 } { 3 } d } \\
{ w _ { B 3 } = w _ { B 4 } = p - \frac { t } { 4 } - \frac { 2 } { 3 } d }
\end{array} \quad \left\{\begin{array}{l}
w_{B 1}=w_{B 2}=p-\frac{t}{4}-\frac{1}{4} d \\
w_{B 3}=w_{B 4}=p-\frac{t}{4}-\frac{3}{4} d
\end{array}\right.\right.
$$

Le différentiel de salaires entre les $\mathrm{A}$ et les $\mathrm{B}$ est inférieur à $d$ dans les entreprises discriminantes grâce à la concurrence des entreprises non discriminantes. Dans les deux cas, le salaire moyen des B est $p-\frac{t}{4}-\frac{1}{2} d$. Par conséquent, le salaire moyen ne fluctue pas selon la répartition des entreprises sur le cercle. Mais le différentiel au sein des travailleurs B est plus élevé lorsque les entreprises identiques sont côte à côte que lorsqu'elles alternent. Dans le premier cas, un B employé par une entreprise non discriminante gagne $\frac{1}{3} d$ de plus que celui employé par une entreprise discriminante contre un écart de $\frac{1}{4} d$ dans le second cas. La répartition des entreprises le long du cercle a donc un effet sur les écarts de salaire au sein de la population discriminée mais n'influence pas le salaire moyen des $B$.

En ce qui concerne la force de travail, des variations sont observées selon l'intensité du goût pour la discrimination, la répartition des entreprises et le nombre d'entreprises sur le marché. Le nombre de salariés A est identique quel que soit l'entreprise et vaut $\frac{1}{4}(1-\gamma)$. Les fluctuations du nombre de salariés sont donc entièrement dues aux B. Dans notre exemple, le nombre de salariés B par entreprise est respectivement :

$$
\left\{\begin{array} { l } 
{ L _ { B 1 } = L _ { B 2 } = \frac { 1 } { 4 } + \frac { 1 } { 3 t } \gamma d } \\
{ L _ { B 3 } = L _ { B 4 } = \frac { 1 } { 4 } - \frac { 1 } { 3 t } \gamma d }
\end{array} \quad \left\{\begin{array}{l}
L_{B 1}=L_{B 2}=\frac{1}{4}+\frac{1}{4 t} \gamma d \\
L_{B 3}=L_{B 4}=\frac{1}{4}-\frac{1}{4 t} \gamma d
\end{array}\right.\right.
$$

Le nombre de salariés est plus faible dans les entreprises discriminantes. Mais il augmente avec la proximité des autres entreprises discriminantes. En effet, lorsque deux entreprises discriminantes sont côte à côte, leur pouvoir sur les B localisés entre elles est plus important. Elles peuvent ainsi offrir un salaire plus faible et attirer un plus grand nombre de B que lorsque leurs voisines sont des entreprises non discriminantes.

De plus, le nombre de salariés dans l'entreprise a un impact sur les profits. En effet, la productivité est un facteur constant donc les entreprises accroissent leurs profits jusqu'à ce que le salaire offert soit trop élevé pour en tirer des bénéfices. Selon la répartition, les profits sont les suivants :

$$
\left\{\begin{array} { c } 
{ \Pi _ { 1 } = \Pi _ { 2 } = \frac { t } { 1 6 } + \gamma d ( \frac { 1 } { 6 } + \frac { d } { 3 t } ) } \\
{ \Pi _ { 3 } = \Pi _ { 4 } = \frac { t } { 1 6 } + \gamma d ( \frac { 1 } { 1 2 } + \frac { 2 d } { 9 t } ) }
\end{array} \quad \left\{\begin{array}{l}
\Pi_{1}=\Pi_{2}=\frac{t}{16}+\gamma d\left(\frac{1}{8}-\frac{d}{16 t}\right) \\
\Pi_{3}=\Pi_{4}=\frac{t}{16}+\gamma d\left(\frac{1}{8}-\frac{3 d}{16 t}\right)
\end{array}\right.\right.
$$

Les profits sont plus élevés pour les entreprises non discriminantes et lorsqu'elles alternent avec des entreprises non discriminantes. Les profits augmentent avec l'intensité du goût pour la discrimination. Les entreprises discriminantes font moins de profits mais il augmente avec la proximité d'entreprises similaires. 


\section{L'INFLUENCE DE LA CONCURRENCE}

Selon Becker, l'impact de la discrimination doit diminuer avec l'amélioration de la concurrence. Dans cette partie, nous allons étudier les différents moyens de faire varier la concurrence dans le modèle et leur impact sur la discrimination.

Une variation de $t$ est équivalente à une variation du degré de concurrence sur le marché du travail. Lorsque $t$ diminue, l'écart de salaire diminue en proportion du salaire total mais reste identique en valeur. Dans le cas spécifique où $t=0$, l'équilibre est en concurrence parfaite et les travailleurs choisissent leur entreprise sans coûts de déplacement. Les travailleurs B décident de travailler pour une entreprise non discriminante et leur salaire reste inférieur du fait d'une demande de travail plus faible que celle des A. La discrimination reste présente sur le marché, mais comme toutes les entreprises ont le même niveau de productivité, les entreprises discriminantes vont disparaître à cause de coûts plus élevés. Sans pouvoir de marché, elles ne pourront se maintenir. Le modèle devient alors un modèle à la Cournot et les différences de salaire entre les A et les B disparaissent comme le prédit Becker.

Lorsque le nombre d'entreprises augmente tout en conservant une répartition uniforme, l'impact des coûts de transports sur le salaire diminue. Les salaires augmentent et se rapprochent de la productivité des travailleurs. En statique, plus le nombre est élevé, plus on s'approche d'une concurrence parfaite.

Lorsqu'une nouvelle entreprise entre sur le marché, l'effet est différent. Les entreprises déjà présentes ont choisi leur position de manière optimale et la nouvelle doit s'insérer entre les entreprises. Supposons qu'une cinquième entreprise désire entrer sur le marché. Elle choisit alors un lieu entre deux entreprises déjà installées (cf figure 2). Selon la répartition des entreprises, ce peut être deux entreprises identiques ou deux entreprises différentes. Dans les deux cas, la distance avec ses voisins est $\frac{1}{8}$ au lieu de $\frac{1}{4}$ pour le reste du cercle. Cela va diminuer les parts de marché de ses voisines directes. Le pouvoir de marché des entreprises déjà présentes et côtoyant directement la nouvelle diminue donc à cause de sa proximité tandis que les deux entreprises qui ne sont pas côte à côte avec la nouvelle ont un plus grand pouvoir de marché et peuvent fixer un salaire plus faible pour leurs travailleurs, quel que soit leur appartenance. Mais l'impact de la discrimination est le même quel que soit le cas : une nouvelle entreprise non discriminante décroît l'impact du goût pour la discrimination sur le marché tandis qu'une nouvelle entreprise discriminante l'augmente.

De plus, la nouvelle entreprise va se placer dans le voisinage d'entreprises discriminantes afin de fixer les salaires les plus faibles possible pour les B. Cela est vrai quelque soit son type : si elle est discriminante, elle peut fixer un salaire plus faible que ses voisines et augmenter l'écart de salaire entre les B. Son utilité est alors plus importante car la désutilité due à son goût pour la discrimination est plus faible que lorsqu'elle est entre deux entreprises non discriminantes. Si l'entreprise n'a pas de goût pour la discrimination, ses profits sont plus importants car le salaire offert aux B dans cette partie du marché est plus faible. Elle peut donc fixer un salaire légèrement plus élevé que ses 


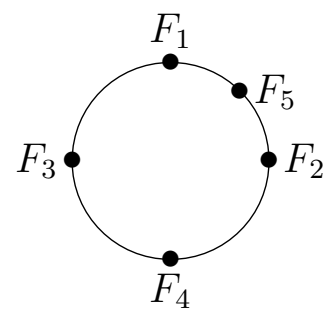

Figure 2: Entrée d'une cinquième entreprise sur le marché.

voisines pour les B et ainsi augmenter sa masse salariale, ce qui augmente également ses profits. L'entreprise bénéficie donc de la présence d'entreprises discriminantes dans son voisinage direct.

\section{CONCLUSION}

Cet article montre qu'un modèle basé sur la discrimination par le goût peut modéliser les faits stylisés concernant la persistance de la discrimination. Il repose sur un pouvoir de marché non négligeable des employeurs sur leurs employés et une hétérogénéité des préférences des travailleurs. Ces hypothèses, proches de ce que l'on peut observer, conduisent à un différentiel de salaire qui ne disparaît pas avec le temps. En effet, les entreprises n'ayant pas goût pour la discrimination font des profits non nuls grâce à la présence d'entreprises discriminantes. Elles n'ont donc aucun intérêt à les évincer du marché par une concurrence agressive. Contrairement à la plupart des modèles de la littérature, il n'y a pas ici de ségrégation des différents groupes de travailleurs au sein des entreprises. Le salaire de la population discriminée n'est pas unique et dépend de la concentration des entreprises discriminantes sur le marché. Il est important de remarquer que la présence d'une seule d'elles entraîne l'existence d'un différentiel de salaire durable entre les deux groupes.

Les entreprises sont supposées identiques afin de simplifier le modèle. Cependant, les travaux empiriques montrent qu'elles sont hétérogènes et ont des niveaux de productivité différents. Dans ce cas, il est naturel d'espérer que les entreprises les plus productives offrent les salaires les plus élevés, ce qui requiert une plus grande force de travail. En étant plus productives, ces entreprises peuvent se permettre de payer des salaires plus élevés pour attirer les salariés supplémentaires dont elle a besoin. Alors les salaires des A suivent une distribution et ne sont plus identiques quel que soit l'entreprise. Les résultats seraient identiques, mais leur lecture serait rendue plus difficile. L'introduction d'hétérogénéité peut donc être une extenssion de cet article.

Une prolongation de ce modèle doit être associée à cet article afin d'étudier les problèmes liés au chômage. En effet, le chômage conduit ici à une situation de monopsone. L'introduction de salaires de réservation hétérogènes permettrait d'introduire le chômage en conservant l'intérêt du modèle. 
ARROW K. J. [1973], The Theory of Discrimination. dans ASHENFELTER, O., \& REES, A. (ed.), Discrimination in Labor Markets, Princeton University Press, p.3-33.

BECKER G. [1957], The Economics of Discrimination, University of Chicago Press.

BHASKAR V., \& TO T. [2003], "Oligopsony and the distribution of wages.", European Economic Review, 47(2), p.371-399.

BHASKAR V., MANNING A., \& TO T. [2002], "Oligopsony and Monopsonistic Competition in Labor Markets.", Journal of Economic Perspectives, 16(2), p.155-174.

BLACK D. A. [1995], "Discrimination in an Equilibrium Search Model.", Journal of Labor Economics, 13(2), p.309-33.

BLACK S.E., \& STRAHAN P.E. [2001] "The Division of Spoils: RentSharing and Discrimination in a Regulated Industry.", American Economic Review, 91(4), p.814-831.

HAMILTON J., THISSE J.-F., \& ZENOU Y. [2000] "Wage Competition with Heterogeneous Workers and Firms.", Journal of Labor Economics, 18(3), p.453-472.

HELLERSTEIN J.K., NEUMARK D., \& TROSKE K.R. [2002] "Market Forces and Sex Discrimination.", The Journal of Human Ressources, 37, p.353-380.

PHELPS E. [1972] "The Statistical Theory of Racism and Sexism.", American Economic Review, 62(4), p.639-651.

SALOP S. C. [1979] "Monopolistic Competition with Outside Goods.", The Bell Journal of Economics, 10(1), p.141-156.

THISSE J.-F., \& ZENOU Y. [1995] "Appariement et concurrence spatiale sur le marché du travail.", Revue Economique, 46(3), p.615-625.

WAUTHY X., \& ZENOU Y. [1999] "Le rôle de l'hétérogénéité des agents sur le marché du travail en concurrence imparfaite.", Revue Economique, 50(5), p.965-984. 